ISSN: $1130-3743$

\title{
DESARROLLO DEL PENSAMIENTO Y PROCESO EDUCATIVO: REFLEXIONES Y ESTRATEGIAS DE OPTIMIZACIÓN CONJUNTA
}

\section{THE DEVELOPMENT OF THINKING AND EDUCATIONAL PROCESS: REFLECTIONS AND STRATEGIES OF JOINT OPTIMIZATION}

\section{MIGUEL A. SANTOS REGO}

Universidad de Santiago de Compostela. Departamento de Teoria e Historia de la Educación. Facultad de Filosofia y Ciencias de la Educación. Campus Universitario Sur. 15706 Santiago de Compostela.

RESUMEN

La educación es proceso de desarrollo humano. Tal desarrollo tiene su epicentro más claro en la destreza por antonomasia: el pensamiento. Partimos de que el gran desafío al que se enfrenta la educación hoy es la necesidad de descubrir una nueva forma de pensamiento. Por ello sugerimos algunos enfoques y direcciones emergentes, intentando ayudar a pensar otras maneras de disponer el proceso educativo; con el objetivo de mejorar los marcos relacionales asociados al éxito dentro y fuera del aula. Entre otras estrategias planteamos los beneficios del aprendizaje cooperativo, el lenguaje global y el aprendizaje holístico o basado en el conocimiento sobre el cerebro.

\section{SUMMARY}

Education it is a process of human development. Such development has its clear epicentre in the skill par excellence: thinking. We start from a main point: 
today, the grat challenge that education has to confront is the need to discover a new way of thinking. Thus, we suggest some emergent approaches and directions, trying to help thinking about other ways to arrange the educational process. In this sense, the objective is to improve the relational frames associted to the success within and out of classrooms. Among other strategies, we reflect about the benefits of cooperative learning, whole language, and holistic learning, also named learning based on brain knowledge.

\section{INTRODUCCIÓN.}

Sin perjuicio de mayor concreción acerca de nuestras intenciones, que no rebasarán el mero ejercicio del intelecto - ayudado por mi corta pericia para el cruce de algunas ideas en torno a cómo plantear el proceso educativo- deseo manifestar que el término "pensamiento" no tiene aquí más referente que el de poder ser entendido como "capacidad o aptitud cognitiva general"; esto es, aquella habilidad global para pensar y razonar que llega a constituir principal baluarte en la relación del sujeto consigo mismo y con el medio en que se sitúa su existencia psico-física.

Tenemos muy claro que la educación es proceso de desarrollo humano. Y siendo así, no puede haber duda de que ese desarrollo tiene su epicentro más inequívoco en la destreza por antonomasia, no otra que el "pensamiento" en su sentido más global y reconocible. Por lo tanto, debemos resistirnos a una mera apelación retórica de la tarea, entre otras cosas, porque la falta de rigor al respecto ha supuesto y sigue suponiendo, por desgracia, poner en peligro algo tan importante como la capacitación del sujeto para elegir y decidir de modo autónomo. Nos remitimos a otro estudio (Santos Rego, 1991) en el que, aparte de resaltar la complejidad del asunto, referíamos algunas de las contradicciones existentes sobre el particular en el mismo sistema educativo, tal vez porque muchas de ellas tienen que ver con valores, actitudes y creencias sociales.

El pensamiento ha sido objeto de mucho estudio en las últimas décadas por parte de la psicología, condicionando una perspectiva hegemónica en su análisis y comprensión. A ello no ha sido ajeno el logro de un mayor conocimiento acerca de cómo generamos, procesamos, almacenamos y recuperamos la información. Esa influencia ha ocasionado, en parte, un olvido tan importante como paradóji$\mathrm{co}$, que aún hoy se pone de manifiesto dentro del sistema educativo. Sencillamente, que el pensamiento es algo inseparablemente unido a la filosofía. Ambas perspectivas son necesarias, obviamente, pero conviene no mezclar las cuestiones y valorar con equilibrio sus potenciales aportaciones al respecto. Recogiendo la útil distinción de Beyer (1990), la psicología se preocupa más por el proceso (el "como" ocurre el pensamiento), y de ahí la lógica de tantos estudios centrados en procedimientos para la mejor enseñanza del pensamiento, mientras que a la filosofía le preocupa la "substancia", esto es, aquello que debe ser incluso en cualquier programa sobre habilidades de pensamiento. 
Se tiene dicho en los últimos tiempos, y no creo que falte razón para ello, que uno de los mayores desafíos, si no el mayor, a los que se enfrenta la educación actual es, sencillamente, la necesidad que tenemos tanto teóricos como profesionales de la educación en general de descubrir, con nuestros alumnos, una nueva forma de pensamiento. Esto y no la tecnología, ni los recursos, ni siquiera el tan mencionado rendimiento de cuentas (en la acepción anglosajona de "accountability") es lo verdaderamente importante, podríamos concluir, si queremos encaminar cualquier esfuerzo educativo con mínimas garantías de éxito en el futuro.

Naturalmente, estaremos de acuerdo con ese amplio aserto si lo que se pretende es señalar una necesidad de búsqueda. Ahora bien, se ha de tratar de una búsqueda que no se agote en la mera exigencia de información más o menos distinta, sino que debe tener como epicentro de sentido un modo nuevo y global de ver el mundo.

La filosofía de la ciencia representa un vasto campo de observación desde el que poder atisbar pasos firmes de avance en el proceso de redefinición. Es por ello que vale la pena, creemos, atreverse a sugerir alguna que otra dirección emergente, con el único fin de intentar ayudar a pensar otras maneras de disponer el proceso educativo, mejorando si cabe el marco relacional entre nosotros y nuestros alumnos. Sobre el particular, reconocemos la ayuda proporcionada por trabajos como los de Crowell (1989) y Beyer (1990), 'estirando, sobre todo, un deseado hilo de continuidad respecto de contribuciones previas (Santos Rego, 1991, 1992, 1995).

\section{CiENCIA Y PENSAMIENTO: SUPERANDO PERSPECTIVAS.}

Fue precisamente un gran filósofo de la ciencia como $A$. Whitebead el que nos abrió aún más los ojos con pruebas de cómo la ciencia nos sigue obligando a cambiar nuestra visión del mundo. Recogiendo su expresión, las viejas bases del pensamiento científico se están volviendo ininteligibles. Es así que conceptos tan asentados como tiempo, espacio, materia, estructura, patrón, función, y otros muchos, precisan de urgente reinterpretación. (cfr. Harris, 1983). Pero no ha sido el único. Otro destacado representante de este ámbito académico como el mismísimo Khun (1957, 1981) alertó vehementemente sobre el hecho de que durante medio siglo hemos estado, casi sin darnos cuenta, en medio de una revolución conceptual que ha ido cambiando la concepción del científico respecto del espacio, la materia, la fuerza, y aún la pretendidamente intocable estructura del universo.

Estamos pasando, o quizás sería mejor decir que ya hemos superado una perspectiva cartesiana del universo - en el que el acento principal se situaba en las partes o elementos- para asumir otra visión, más configuracional, con énfasis expresivo en todos y patterns reguladores (Drucker, 1969). De modo y manera que hemos puesto en entredicho cualquier línea divisoria entre áreas de estudio o de conocimiento.

La realidad es que tales mutaciones históricas en el devenir del pensamiento hace que la conceptualización del mundo propia del entramado cultural en el 
que nos movemos disminuya su consistencia en relación con el conocimiento científico y, consecuentemente, los modelos conceptualizadores al uso común se vuelven cada vez menos útiles para explicar ese mismo mundo. Esto es, sirviendo como ejemplo, el mundo cartesiano-newtoniano, caracterizable según notas de orden, secuencia lineal y predicción mecanicista solo es válido en contextos limitados. No han faltado científicos en manifestar su convencimiento de que lo que hemos hecho desde la época del gran Isaac Newton ha sido visualizar nuestro mundo o bien como una máquina estéril o bien como un estado de entropía y desmoronamiento. Davies (1988) parte justamente de esa posición y expone un nuevo paradigma que intenta reflejar un punto de vista ciertamente más integrador, abarcando los aspectos colectivos, cooperativos y organizativos de la naturaleza.

Si nos atrevemos a entrar en el apartado de implicaciones posibles, tal vez convenga hacernos una pregunta del tipo ¿qué puede causar esos cambios de pensamiento en una cultura? Lo más razonable, creo, es contestar diciendo que su origen está vinculado a nuevos modelos de comprensión del mundo, ya que tales marcos comprensivos se demuestran inconsistentes con las descripciones emergentes de la realidad. Como sabemos, es lo que pasa en la ciencia cuando los nuevos datos empíricos hacen añicos un marco establecido o cuando se aceptan, a la vista de sus postulados, nuevas proposiciones teóricas. Pero sucede incluso algo más. Puesto que esos cambios en el pensamiento científico se van filtrando paulatinamente a través de distintas agencias sociales, al final son las propias creencias y valores los que resultan alterados. Recordemos, sin ir más lejos, la espectacular implicación derivada del abandono de la creencia en la tierra como centro del universo.

\section{El CONOCIMIENTO COMO DESAFÍO DE CREENCIAS ACEPTADAS.}

Con lo que acabamos de decir, sería ingenuo dejar de reconocer que el pensamiento científico no afecta a la sociedad y también - faltaría más- a la educación como fenómeno y proceso. En pocas palabras, el nuevo conocimiento desafía continuamente creencias educativas aceptadas, algunas de las cuales son perfectamente resumibles del modo que sigue (si bien sería inabordable el número de ejemplificaciones al respecto):

a) ¿Cuántos educadores creen, realmente, tomando como base su experiencia y capacidad de observación, que una persona aprende a leer mediante el dominio secuencial de destrezas (entre 80 y 125) en un continuum lector?

b) ¿Cuántos educadores mantienen aún hoy que la enseñanza efectiva consiste en la simple suma de cinco o siete sets de conductas organizadas idealmente en un orden lineal?

c) ¿Puede creer alguien realmente que los tests miden aquello que conocemos?

d) ¿Puede reducirse el conocimiento a materias separadas que sólo raramente tienen relación con la realidad experiencial? 
Puede que sea suficiente para mostrar una evidencia bastante clara, a saber, las inadecuaciones de los puntos de vista cartesiano-newtonianos tanto en la ciencia en general como en la ciencia de la educación en particular.

Por otra parte, se menciona frecuentemente, y con razón, que una concepción tremendamente obsoleta se hace palpable en la forma en que fragmentamos la realidad. Pues bien, los avances experimentados en las últimas décadas en todos los campos de la ciencia, incluyendo naturalmente a las ciencias sociales, indican justamente que el universo no es, ni representa, una multiplicidad mecánica de cosas sino que se configura como única y dinámica unidad.

Sin embargo, aún en la actualidad, una gran parte de nuestras asunciones sobre la naturaleza de la realidad hace que reduzcamos las cosas según partes discretas, favoreciendo una visión de nosotros mismos como entes separados del medio. Si esto es así no pocas veces, mencionemos el peligro que salta a la vista: cualquier perspectiva transmitida al alumno, sobre todo de niveles iniciales y medios de escolaridad, en la dirección de que el universo está compuesto de fragmentos independientes, servirá para reproducirla, casi sin remedio.

No seamos mojigatos y admitamos con sentido crítico que buena parte del curriculum y otra nada despreciable de nuestras prácticas de enseñanza (instructivas) sugieren a los alumnos, como diría Bohm (1980) que, al menos en principio, el sujeto que piensa está absolutamente separado de la realidad en la que está pensando. Y lo peor es que responde bastante bien a lo que sucede en muchas escuelas. Efectivamente, encontramos materias separadas, destrezas separadas, objetivos separados, evaluaciones separadas, métodos lineales, continuums segmentados, técnicas conductuales, etc., sin mencionar el caso de tantas aulas aisladas que por ahí y por aquí existen.

No se trata, ahora, tanto de discursear acerca del enfoque errado que se desliza en la realización de tales prácticas, como de afirmar que se basan en supuestos que tienen cada vez menos que ver con las nuevas ideas y concepciones que vamos asumiendo sobre el mundo. Y con el problema añadido de que sirven para comunicar a los alumnos un mundo de conocimiento exento de significatividad, junto con un mundo en el que el resultado es independiente del proceso.

Siempre hay una pregunta en el seno de la escuela, incluso de la comunidad, al tratar estas cuestiones en prospectiva. Sencillamente, ¿qué deberían saber nuestros alumnos en el futuro? La sugerencia implícita, para una respuesta educativa, apunta a una consideración, en términos de igualdad, de contenidos y procesos. Los primeros son caracterizados normalmente como "información", mientras los segundos aparecen más unidos a lo que llamamos "método".

Convencionalmente es así como se tocan los términos de "contenido" y de "proceso". Con este nuevo marco de comprensión, que obliga a reconceptualizarlos en sintonía con sus proposiciones básicas, la forma de entenderlos varía sustancialmente. No es que se relacionen el uno con el otro sino que cada uno está incorporado en el otro. Respecto del contenido, el énfasis se pone ahora en el significado o, si se prefiere, en las preguntas que están detrás de la información, en los aspectos estructurales de las disciplinas, así como en las relaciones que el contenido específico de un campo tiene con el contenido específico de otro campo. 
El proceso, por su parte, ya no es únicamente "método". Es aprendizaje centrado en la "manipulación" de contenidos con objeto de proceder a la ampliación de relaciones. Se trata, más bien, de una exploración de las formas en que aprendemos e internalizamos los contenidos, y también del modo en que aplicamos un contenido para producir conocimiento significativo del mundo y de nosotros mismos.

Tampoco sobre al propósito del trabajo hacer explícita una cuestión que a menudo aparece en nuestro verbo explicativo de cuanto nos rodea. Se trata de la apelación a la complejidad del mundo, al que se llega a tildar ya de caótico. No es infrecuente que cuando se les pide a los alumnos una descripción de su mundo surjan palabras del tipo "complejo", "dinámico", "estresante", "acelerado", etc. Lo curioso del asunto es la similitud del lenguaje, figurativamente hablando, que están usando bastantes científicos e investigadores: palabras como "azaroso", "interactivo", "dinámico", "sinérgico", ilustran la proximidad. Todos recordamos seguramente que nada menos que el Profesor Ilya Prigonine, Premio Nobel de Química, acrecentó su celebridad con un libro que tituló Order out of Chaos (1984). Del mismo modo, puede que recordemos la descripción que por aquellas fechas hacía Donald Schon de los directores de grandes consorcios como "managers" del caos.

Pero, con todo, algo tenemos por bastante seguro. Y es que, a pesar de la multitud de interacciones, pretendidamente aleatorias, que tienen lugar en el mundo, contamos con propositividad y unidad (que de cuando en vez parecen incluso desafiar la explicación). Lo vemos más diáfano si tenemos en cuenta la física sub-atómica o el conocimiento del cosmos que se debe al astrónomo.

Breve y sencillamente: los humanos no estamos separados de la unidad, formamos parte de ella. Para decirlo en lenguaje metafórico, no podemos percibirnos a nosotros mismos como observadores independientes parados al lado de una corriente rápida, anotando sus giros y vueltas, estudiándola objetivamente. No podemos conceptualizar el flujo como algo que navega desde un punto $\mathrm{A}$ a un punto B; es preciso que añadamos una nueva relación para el flujo, porque, como diría Jautsch (1975), "nosotros somos el flujo". Con ello lo que deseamos no es más que resaltar una fusión entre objetividad y subjetividad al objeto de intentar un enfoque que abarque la unidad de ambas.

\section{4. ¿QUÉ haY PARA ESto? Algunas ESTRATEgias DE INTERVENCIÓN PEDAGÓGICA.}

Desde la filosofía que procuramos resumir hasta el momento, queremos mencionar seguidamente determinados enfoques de desarrollo que reflejan aspectos latentes en los cambios habidos en el pensamiento científico y social. No nos es posible ofrecer aquí más que un bosquejo de sus potencialidades en la dinámica de metas educativas convergentes con la estimulación del pensamiento en las aulas.

El primero que nos gustaría destacar es el llamado "Aprendizaje Cooperativo" (Cooperative Learning), uno de los que más atención ha recibido en los últimos años alrededor del planeta. A estas alturas es bien conocido el éxito que se consigue cuando los alumnos, reunidos en pequeños grupos, aprenden contenidos de 
modo cooperativo. Esto no quiere decir que estemos enfatizando algo (las prácticas de grupo o agrupamientos) poco novedoso en educación.

De lo que se trata es de significar la inequívoca importancia de los aspectos cooperativos del proceso. Es así como cada alumno se convierte en responsable no sólo de su propio aprendizaje sino también del aprendizaje de cada miembro del grupo. La imposible pretensión de exhaustividad en el análisis del aprendizaje cooperativo, para lo cual remito a ciertas referencias básicas (Santos Rego, 1990; Slavin, 1991, 1995), obliga a una relación apretada de notas como:

- Potencia el crecimiento cognitivo y afectivo del alumno al procurar nuevos escenarios de socialización escolar.

- Estimula la auto-regulación en el proceso de aprendizaje individual. Se ha comprobado que a medida que los alumnos intercambian verbalmente ideas durante la realización de tareas cooperativas optimizan el uso de un vocabulario específico que dirige y controla la conducta de resolución de problemas. Lo verdaderamente importante es que ese vocabulario activado en el proceso de resolución se va internalizando progresivamente y da lugar a lo que Vygotsky llamó "habla interna", destreza cognitiva de enorme significado para el desarrollo del pensamiento humano.

- Permite el desarrollo y consolidación de distintos tipos de habilidades y destrezas sociales.

- Favorece el desempeño y/o rotación de roles, con lo que se aumenta la participación activa del alumno en su propio proceso de aprendizaje. Además la tutoría de iguales (peer tutoring), indisociable del aprendizaje cooperativo bien diseñado y aplicado, permite no sólo un intercambio de roles sino la movilización de procesos y sub-procesos (de comprensión, razonamiento, percepción, representación, etc.) indispensables para el aprendizaje y la configuración del sujeto.

- Los resultados no se consideran exclusivamente desde un prisma individual, sino que se valora, además, la contribución al grupo, entendido como comunidad de aprendizaje.

- El éxito del grupo, consiguientemente, dependerá de su funcionamiento o actuación resultante, donde la ayuda mutua y la solidaridad intergrupal adquieren toda su relevancia.

- El máximo acento, como es lógico, se coloca en la interacción de los alumnos y en las prácticas experienciales que tienen lugar cuando aprenden juntos.

Se alienta, en definitiva, la conexión entre los alumnos, poniendo de relieve el rol de una interacción no lineal en la resolución de problemas. La significación emocional de ser un miembro estimado del grupo se relaciona así con el proceso de aprendizaje. Y el contenido pasa a ser considerado no sólo como información sino también como experiencia de cooperación en si mismo.

Oportuna es, igualmente, la mención de otra estrategia bautizada como "Aprendizaje Conectado" (Complex Instruction). Viene a ser una variante del agrupamiento cooperativo en el que, sintéticamente, cobra auge el rol del profesor en el proceso de dirección. Los grupos son dispuestos para el trabajo mediante acti- 
vidades de descubrimiento, con expectativas de resultados a nivel individual y grupal. Se asignan los correspondientes roles a cada miembro, al tiempo que se esperan, fundamentalmente, resultados grupales. A diferencia de lo que es más corriente en la perspectiva del aprendizaje cooperativo, aquí se pone un mayor énfasis en el contenido de la lección.

Permitaseme una breve descripción que ilustre el "modus operandi": una media de seis grupos exploran diferentes actividades que se relacionan con distintos aspectos del mismo concepto. Cada día la lección incluye una introducción, actividad de grupo, y una cobertura que implica a los grupos en una dinámica de participación (lo básico es compartir) mutua en torno a los descubrimientos. A lo largo de seis días los grupos intercambian actividades, ampliando sus interrelaciones.

La técnica proporciona, en términos globales, una firme conexión entre contenido y proceso. Teóricamente somos conscientes de la importancia adquirida, respecto del aprendizaje, por las interrelaciones entre sociología, psicología, paradigma interactivo y contenido curricular. Esos marcos disciplinares se hacen más provechosos una vez se han desarrollado y acentuado tales interconexiones.

Otra estrategia en la misma línea de acción recibe el nombre de "Lenguaje Global" (Whole Language). Se trata de un enfoque de lenguaje y aprendizaje de destrezas instrumentales (literacy) en el que se rechaza de plano la separación convencional de los procesos que afectan al lenguaje dentro de la lectura, la escritura, el habla y la escucha. En su lugar, el lenguaje se concibe como una totalidad, constituyendo una parte integral de nuestra experiencia, así como un medio natural de expresión. De ahí que, sin sorpresa alguna, la literatura, el arte, la música y la expresión dramática se conviertan en cauces interesantes para estimular este tipo de expresión.

Abundando un poco más en el sentido de la estrategia, digamos que también contribuye a enseñar el proceso inherente a la escritura, haciendo que los alumnos se conviertan en autores de su propias obras, de tipología variada.

Curiosamente, los errores se consideran fuentes estimables de información que sirven para ayudar a los alumnos a lograr mayores grados de alfabetización. Complementariamente, se acentúa el significado y la experiencia a medida que los niños se van implicando en este proceso que les llevará a convertirse en productores de significado. Tal vez por eso se considera al "Lenguaje Global" como magnífico ejemplo de bastantes conceptos paradigmáticos nuevos. No en vano son conceptos centrales del enfoque algunos como "integración", "complejidad" y "holismo". Así, proceso y contenido resultan interconectados, del mismo modo que lo llegan a estar el alumno y el aprendizaje.

Tampoco conviene olvidar la enorme relevancia adquirida por estrategias derivadas del impacto que han tenido las implicaciones educativas de la investigación sobre la hemisfericidad cerebral, origen de un enfoque holístico en la educación y que algunos han llamado "aprendizaje basado en el cerebro" (brain-base learning) y otros simplemente "pedagogía adaptativa" (ver Caine y Caine, 1991; Doval, Santos, Barreiro, y Crespo, 1993; Doval Salgado y Santos Rego, 1996, en prensa; Sonnier, 1989, 1992).

Se trata de un enfoque global que incluye un número de técnicas basadas en el nuevo conocimiento acerca del funcionamiento cerebral, al que contribuye 
enormemente la investigación en el campo de la neurociencia. Dado que es imposible ocuparnos con amplitud de esta perspectiva, resumiremos los más significativos principios que la informan, esperando que sean ellos mismos los que ayuden a reflejar sus auténticas posibilidades para una teoría del aprendizaje en el marco conceptual que ocupa nuestras reflexiones. Serían estos:

- Si bien solo es posible centrar nuestra atención momentánea en un aspecto o cuestión, el cerebro, sin embargo, procesa y organiza muchas cosas al mismo tiempo.

- El aprendizaje supone una experiencia psicológica que es mucho más que un simple ejercicio mental.

- El cerebro organiza el nuevo conocimiento sobre la base de la experiencia y el significado previos.

- Son los mismos patrones (patterns) derivados de la experiencia los que ayudan a determinar la significación del contenido.

- Entre el estado emocional de un sujeto y su aprendizaje se produce una relación fundamental y crítica.

- En relación con lo dicho anteriormente, el cerebro procesa partes y todos de modo simultáneo.

- Los estímulos periféricos son procesados por el cerebro consciente e inconscientemente.

- Tenemos un sistema de memoria espacial que recupera la experiencia rápida y fácilmente. Y es justamente esta memoria espacial la que nos permite recordar imágenes holísticas. Sobre el particular, y dado cualquier evento cotidiano próximo, por más que no hagamos intento alguno de memorizar elementos concretos, nuestra memoria acerca de esa experiencia permanece intacta y es bastante pormenorizada.

- Hechos o destrezas no incorporadas en la memoria espacial necesitan más práctica.

- El cerebro responde ante cualquier desafío, pero puede decirse que su efectividad se atenúa en situaciones de amenaza.

En una perspectiva de intervención pedagógica, el enfoque de aprendizaje "basado en el cerebro", en considerable armonía con sus homólogos "holístico" y "adaptativo" depende, para alcanzar cotas suficientes de éxito práctico, de la sensibilidad del docente en cuanto a un reconocimiento básico: el de la unidimensionalidad de su tendencia cognitiva, condicionada por su peculiar modo de percibir y procesar la información. El profesor precisa de conciencia clara para flexibilizar su modo de pensar y actuar adaptándolo a las exigencias de estilo perceptivo-cognitivo de sus alumnos. En otras palabras, aquel alumno al que no estimula y entrena, pluridimensionalmente, ambos hemisferios (analítico/auditivo y global/visual) ve aumentada la posibilidad de atrofia de su potencial de aprendizaje. Esto no significa que la acción pedagógica deba tender hacia un puro ambidextrismo hemisférico, sino que dominancia y subalternación hemisférica se hallen en la proporción debida (ver Doval, Santos, Barreiro y Crespo, 1993). 
Por el relatorio de principios que hemos presentado estamos en condiciones, creemos, de advertir la compatibilidad y coherencia que manifiestan con la forma de conceptualizar el mundo inherente al planteamiento hecho en este trabajo, sintetizable bajo las notas de complejidad, unidad e interrelacionabilidad, entre otras.

\section{Más alLá de las prácticas pedaGóGicas}

Naturalmente, no se agotan en tales estrategias las respuestas a los múltiples desafíos que tenemos delante. Aspirar al éxito en el impulso hacia nuevas formas de pensar, exige la ubicación del foco atencional más allá de las prácticas pedagógicas. Lo verdaderamente importante es modificar o alterar la estructura de la escuela, ya que esta, en si misma, no puede imaginarse separada del aprendizaje. No sorprenda, por tanto, el acento en una consideración de la escuela alejada de aquellas que la conciben como simple lugar o mera organización. Porque, sin rodeos, la escuela es enseñanza y aprendizaje.

Entonces, en lugar de tenerlas como instituciones para aprender, lo bueno sería deslindar vías como para poder hacer de las escuelas instituciones o, simplemente, espacios de aprendizaje.

Convendremos sin ambages que el aislamiento no puede conducir a aprendizajes con verdadero significado, y más si lo situamos en un contexto organizacional. Pero, desafortunadamente, ¿cómo negar que las escuelas aún siguen aislando a los niños de la experiencia? O, ¿cómo dejar de reconocer que las escuelas están aisladas entre sí muchas veces? De la misma forma que muchos profesores siguen estando aislados en las aulas, o que muchos directores y administradores están aislados respecto de profesores, alumnos y otros directores y administradores. En definitiva, que el proceso de evaluación del personal (staff) continúa desarrollándose al margen de un auténtico desarrollo profesional. Algo parecido podríamos señalar en cuanto al desarrollo de destrezas fundamentales. Y por ello la cuestión permanece centrada en los modos de hacer que la escuela y sus partes constituyentes se vuelvan más integradas y cohesionadas.

Es evidente también que otro de los desafíos a nuestro pensamiento se referencia en el propio curriculum. Han sido toda clase de estudios interdisciplinares los que han hecho saltar a la vista que cada disciplina tiene vínculos y lazos de unión con otras. Por lo tanto, si lo que buscamos es una educación integrada y una enseñanza suficientemente inter/transdisciplinar, nos debemos obligar a la comunicación de significados imbricados en actividades y contenidos propuestos. Así, tendríamos que la consideración real de relaciones y de patrones es lo que puede disminuir el aislamiento y lo que puede potenciar una integración significativa. Porque, entre otras cosas, los temas que seleccionamos son organizadores útiles cuando ayudan a examinar la unidad básica inherente a la mayor parte de los conceptos. Percatémonos simplemente que el estudio de los macro-fenómenos, tipo guerra, paz, asuntos medio-ambientales, dilemas políticos, etc. puede resultar beneficiado por estudios transdisciplinares si logramos adoptar un pensamiento no reduccionista. 
El proceso de creación de nuevas formas de pensamiento dentro de una cultura encuentra uno de sus problemas no menores en el desarrollo de metáforas apropiadas que contribuyan a una transición exenta de peligros añadidos. Ahora bien, hay que tener presente que las metáforas no son explicaciones propiamente dichas. Se las puede entender, más bien, como guías que proporcionan zonas para la expansión del pensamiento.

Pensando en metáforas que tal vez puedan ayudar a ocuparnos de conceptos en principio abstractos en la vida cotidiana, encontramos, por ejemplo, un concepto que, sobre todo en su acepción inglesa, constituye una metáfora útil. Hablamos del concepto de "incorporabilidad" (embeddedness). Incorporado en la enseñanza está el aprendizaje (¿o es posible tratarlos de forma separada?. Igualmente, incorporado al concepto de maestro está el de alumno, ya que uno resulta incompleto sin el otro. Lo mismo que incorporado en la historia está el arte, la ciencia, la literatura, la música, la tradición, etc. O que en el trabajo, por no decir más, está incorporado el juego.

Es bien claro que conceptos opuestos y duales se vuelven insignificantes si los analizamos en sentido metafórico. Así, tenemos el caso de paradojas que miméticamente respecto de una lógica "occidental", se tenían por "ilógicas" en el pasado están siendo objeto de atención en estos momentos. Es lo que ocurría, por ejemplo, con la luz, de la cual se decía que era o bien una partícula o bien una onda (aquella lógica no admitía que pudiera ser ambas). Sin embargo, hoy sabemos que la luz es tanto una partícula como una onda. Es por lo que concluimos que aquello que incorporamos o asociamos a las personas, cosas, organizaciones, curriculum, o prácticas pedagógicas no es fácil de desentrañar, pero expresa la esencia de toda una visión del mundo emergente.

Reparemos en otra metáfora con visos de solidez como puede ser la visión de la educación como arte. Admitido que no es esta una metáfora muy novedosa es posible sacarla a colación debido a razones suficientemente singulares. Ante todo, incorporada en la noción de arte tenemos la técnica, la imaginación, la filosofía, la perspectiva, la experimentación y, por supuesto, el "ojo interno" del mismo artista.

Dicho brevemente, la gama de los medios está tan abierta como la combinación de los propios medios. El dominio, por tanto, puede darse a niveles muy diferentes:

- pasado, presente y futuro están interconectados.

- proceso y producto están unidos, esto es, nunca los podemos plantear como realmente independientes.

- el arte no es sólo un asunto estético, sino que también puede ser social o político. No en vano se relaciona significativamente con la experiencia humana. Y obliga, de hecho, a una permanente reinterpretación y re-creación del mundo.

Si ello es cierto, y así lo pensamos, el arte — dada su comprensión metafórica para la educación - manifiesta un considerable potencial de ayuda para que podamos crear, y valorar, una nueva visión de la escuela en nuestro espacio vital. 
Es bastante reprobable, que las artes no hayan merecido más asignación de tiempo en los curricula derivados de los planes de reforma educativa en muchos países, incluido el nuestro. Sin embargo, se sigue insistiendo en que una de las metas es, vaya con la ironía, el desarrollo de la imaginación y de la sensibilidad. Como ha dicho Eissner (1992), las artes pueden ayudar al alumno a descubrir problemas no restringidos a los que tienen una sola respuesta correcta, a darse cuenta de la interacción entre forma y contenido, y a advertir que la flexibilidad en los objetivos es señal de una inteligencia fluida capaz de hacer frente a las impredecibles vicisitudes de la vida.

\section{UNA CONCLUSIÓN EN PERSPECTIVA.}

Con todo lo que hemos referido, terminamos este continuum reflexivo sugiriendo una recapitulación que puede incluir una visión del mundo con cabida para cuatro perspectivas genéricas:

a) el pasado constante; es decir, justamente aquello que permanecerá como parte de nuestro previsible futuro.

b) el pasado que desaparece: instituciones, tradiciones, incluso valores; en definitiva, lo que seguramente está en franco declive pero que continuará afectando a la vida de mujeres y hombres.

c) el pasado continuo: incluyéndose a cada uno de nosotros y de nuestros jóvenes, junto a estructuras, ideas y valores que se adoptaron en el pasado y que, con casi total seguridad, nos acompañarán en el futuro.

d) el futuro creado: sencillamente, los actos espontáneos de creatividad que proporcionan algo de forma y dirección al mundo que se nos anuncia y que, en parte, ya está con nosotros (cfr. Crowell, 1989).

Tendremos que esmerar, entonces, la tarea de ayudar a que los alumnos consigan explorar con tino su futuro, porque estaremos explorando también el que nos ha de corresponder como humanos. Pero debe dejarse absolutamente claro que el desafío que puede representar una nueva forma de pensar no es ninguna concesión proclive al abandono de valores que supongan significado en la acción comunicativa. El desafío es de participación en la creación de una nueva visión del rol que hemos de asumir como seres humanos comprometidos con una educación que sirva para desarrollar el potencial que encierra cada sujeto. Tarea harto difícil si no ponemos empeño en modificar a la baja la tremenda carga de programación lineal que sigue caracterizando al proceso de enseñanza-aprendizaje en gran parte de las escuelas que nos circundan.

Reitero, pues, algo ya proclamado como vía de salida progresiva: la estimulación del pensamiento conectado a la actividad educativa y/o escolar pasa por la consideración del aula como "comunidad de indagación" (Santos Rego, 1991) y por una reconceptualización en regla del papel que ha de jugar el profesor en todo el proceso. Creo que la dirección adecuada puede quedar un poco más despejada asumiendo estrategias consonantes con las que aquí se han esbozado. 
MIGUEL A. SANTOS REGO

DESARROLLO DEL PENSAMIENTO Y PROCESO EDUCATIVO

BiBLIOGRAFÍA

BEYER, B.K. (1990): What philosophy offers to the teaching of thinking, Educational Leadeship, 47, 5, pp. 55-60.

Boнm, D. (1980): Wholeness and the implicate order (New York, Routledge and Kegan Paul).

CAINE, R.N. y CAINE, G. (1991): Making connections: teaching and the human brain (Alexandria, Virginia: A.S.C.D.).

Crowell, S. (1989): A new way of thinking: the challenge of the future, Educational Leadershipi, 47, 1, pp. 60-63.

DAVIS, P. (1988): The cosmic blueprint: new discoveries in nature's creative ability to order the universe (New York, Simon and Schuster).

Doval Salgado, L. y Santos Rego, M. A. (1996): De la educación holística a la pedagogía adaptativa, en Doval, L. y SANTOS, M. A. (eds): Neurociencia y Educación, Universidad de Santiago de Compostela (en prensa).

Doval, L.; SANTOS, M. A.; BarReiro, F.J., CRespo, A. (1993): Estilos docentes y discentes: consideraciones pedagógicas a la luz de la neurociencia, Revista Española de Pedagogía, 195, pp. 311-323.

DRUCKer, P. (1969): The age of discontinuity (New York, Harper nad Row).

EISSNER, E.W. (1992): La incomprendida función de las artes en el desarrollo humano, Revista Española de Pedagogía, 191, pp. 15-34.

HARRIS, P. (1983): New world, new ways, new management (New York, Amacom).

Khun, T. (1957): The copernican revolution (Cambridge, Mass., Harvard University Press).

KHUn, T. (1981): La estructura de las revoluciones científicas (México, Fondo de Cultura Económica).

JANTSCH, E. (1975): Design for evolution (New York, Braziller).

SANTOS Rego, M.A. (1990): Estructuras de aprendizaje y métodos cooperativos en educación, Revista Española de Pedagogía, 190, pp. 53-78.

SAntos Rego, M. A. (1991): Por una pedagogía del pensamiento ante la coyuntura de reforma, PAD' E, 1, 2, pp. 165-182.

SANTOs Rego, M. A. (1992): La acción reflexiva en educación: buscando lo sustancial, Revista de Teoría de la Educación, IV, pp. 91-112.

Santos Rego, M. A. (1995): Pensar e aprender: o cerne dunha nova cultura na escola, Revista Galega do Ensino, 6, 59-67.

Schon, D. (1984): The reflective practitioner. How professionals think in action (New York, Basic Books).

SLAVIN, R.E. (1991): Synthesis of research on cooperative learning, Educational Leadership, 48, 5, pp. 71-82.

Slavin, R.E. (1995): Cooperative learning (2nd. ed.) (Boston, Allyn and Bacon).

SONNIER, I.L. (1989): Affective education: methods and techniques (Englewood Cliffs, N.J., Educational Technology Publications).

SONNIER, I.L. (1992): Hemisphericity as a key to understanding individual differences (Springfield, II., Charles C. Thomas Publisher). 